\title{
Bad Communities with High Modularity
}

\author{
Ath. Kehagias and L. Pitsoulis \\ Faculty of Engineering \\ Aristotle Univ. of Thessaloniki \\ kehagiat@auth.gr, pitsoulis@auth.gr
}

April 24, 2022

\begin{abstract}
In this paper we discuss some problematic aspects of Newman's modularity function $Q_{N}$. Given a graph $G$, the modularity of $G$ can be written as $Q_{N}=Q_{f}-Q_{0}$, where $Q_{f}$ is the intracluster edge fraction of $G$ and $Q_{0}$ is the expected intracluster edge fraction of the null model, i.e., a randomly connected graph with same expected degree distribution as $G$. It follows that the maximization of $Q_{N}$ must accomodate two factors pulling in opposite directions: $Q_{f}$ favors a small number of clusters and $Q_{0}$ favors many balanced (i.e., with approximately equal degrees) clusters. In certain cases the $Q_{0}$ term can cause overestimation of the true cluster number; this is the opposite of the well-known underestimation effect caused by the "resolution limit" of modularity. We illustrate the overestimation effect by constructing families of graphs with a "natural" community structure which, however, does not maximize modularity. In fact, we prove that we can always find a graph $G$ with a "natural clustering" V of $G$ and another, balanced clustering $\mathbf{U}$ of $G$ such that (i) the pair $(G, \mathbf{U})$ has higher modularity than $(G, \mathbf{V})$ and (ii) $\mathbf{V}$ and $\mathbf{U}$ are arbitrarily different.
\end{abstract}

\section{Introduction}

This paper describes some problems which may arise in using Newman's modularity function $Q_{N}$ for community detection. Modularity is one of the most popular quality functions in the community detection literature. It is not only used to evaluate the community structure of a graph, but also to perform community detection by modularity maximization. However, it is well known that modularity maximization can, in certain cases, yield the "wrong" community decomposition. Previous work on this aspect has focused on the modularity resolution limit, which causes underestimation of the true number of communities. In this paper we focus on the opposite effect, in other words we show that, in certain cases, modularity maximization can overestimate the number of communities.

In Section 2 we present our nomenclature and notation; let us stress from the beginning that we will use "cluster" as a synonym of "community" and "clustering" to denote both a partition of the nodes of a graph and the activity of creating such a partition.

In Section 3 we present an interpretation of $Q_{N}$ which, as far as we know, has not been discussed previously. It is well known that the modularity of a graph $G$ can be written in the 
form $Q_{N}=Q_{f}-Q_{0}$, where $Q_{f}$ is the intracluster edge fraction of $G$ and $Q_{0}$ is the expected intracluster edge fraction of the null model, i.e., a graph $G^{\prime}$ which has the same expected degree distribution as $G$ but randomly distributed edges. As explained in Section 3.2, maximization of $Q_{f}$ favors clusterings with a small number of clusters and few edges across clusters. On the other hand, as explained in Section 3.3, minimization of $Q_{0}$ favors clusterings with a large number of clusters and each cluster having approximately equal degree (we call these "balanced clusterings"). Cluster number selection is performed by balancing these two opposite effects in the maximization of $Q_{N}$.

In Section 4.1 we exploit the behavior of $Q_{0}$ and construct examples in which modularity maximization yields arbitrarily inaccurate clusterings. More specifically, we construct a class of graphs $G_{K, N_{1}, N_{2} K}$ (where $K, N_{1}, N_{2}$ are parameters of the graph) with the following properties.

1. Each graph $G_{K, N_{1}, N_{2}}$ has a "natural" clustering $\mathbf{V}_{K, N_{1}, N_{2}}$ (which, however, does not maximize modularity).

2. We can find graphs $G_{K, N_{1}, N_{2}}$ and clusterings $\mathbf{U}_{K, N_{1}, N_{2}, J}$ such that, by appropriate selection of $K, N_{1}, N_{2}$ and $J$, the following hold:

2.1. The pair $\left(G_{K, N_{1}, N_{2}}, \mathbf{U}_{K, N_{1}, N_{2}, J}\right)$ has higher modularity than the pair $\left(G_{K, N_{1}, N_{2}}, \mathbf{V}_{K, N_{1}, N_{2}}\right)$;

2.2. The modularity of $\left(G_{K, N_{1}, N_{2}}, \mathbf{U}_{K, N_{1}, N_{2}, J}\right)$ can become (by appropriate selection of $J$ ) arbitrarily close to one;

2.3. the Jaccard similarity between clusterings $\mathbf{V}_{K, N_{1}, N_{2}}$ and $\mathbf{U}_{K, N_{1}, N_{2}, J}$ can become (by appropriate selection of $J$ ) arbitrarily close to zero (hence $\mathbf{V}_{K, N_{1}, N_{2}}$ and $\mathbf{U}_{K, N_{1}, N_{2}, J}$ are arbitrarily different in the Jaccard sense).

We prove similar results for another class of graphs in Section 4.2 .

In Section 5 we discuss the implications of our results and (previously published) related work by other authors. It is often claimed that community detection by modularity maximization should be preferred over other community detection methods because it does not require knowing the number of clusters in advance. However, in light of our results (as well as the previously known modularity resolution limit) this claim appears ill-founded. We conclude the paper with a discussion of alternative cluster number selection methods.

\section{Preliminaries}

1. A graph $G$ is a pair $(V, E)$, where $V$ is the node set (we will always assume $V=\{1,2, \ldots, n\}$; hence the number of nodes is $n=|V|$ ) and $E \subseteq\{\{u, v\}: u, v \in V\}$ is the edge set (and $m=|E|$ is the number of edges). Hence we will deal with finite graphs without multiple edges and loops.

2. The adjacency matrix of $G$ is an $n \times n$ matrix $A$ with $A_{u, v}=1$ iff $\{u, v\} \in E$ and 0 otherwise. There is a one-to-one correspondence between a graph $G$ and its adjacency matrix $A$. 
3. A clustering of $G=(V, E)$ is a partition $\mathbf{V}=\left\{V_{1}, \ldots, V_{K}\right\}$ of $V$. The clusters are the node sets $V_{1}, \ldots, V_{K}$, which satisfy $\cup_{k=1}^{K} V_{k}=V$ and $\forall k, l: V_{k} \cap V_{l}=\emptyset$. The size of the clustering is $K$, the number of clusters. Given a graph $G=(V, E)$, we denote by $\mathcal{V}$ the set of all clusterings of $V$ and by $\mathcal{V}_{K}$ the set of clusterings of size $K$. Sometimes we call $V_{k}$ a community; this is simply a synonym of "cluster".

4. Given a clustering $\mathbf{V}=\left\{V_{1}, \ldots, V_{K}\right\}$ of the graph $G=(V, E)$, we define the following edge sets (for $k=1, \ldots, K)$ :

$$
E_{k}=\left\{\{u, v\}: u, v \in V_{k} \text { and }\{u, v\} \in E\right\}
$$

i.e., $E_{k}$ is the set of edges with both ends being nodes of $V_{k}$. The edges contained in $\cup_{k=1}^{K} E_{k}$ are the intracluster edges; the remaining edges, i.e., the ones contained in $E-$ $\cup_{k=1}^{K} E_{k}$ are the extracluster edges.

5. The degree function $\operatorname{deg}(\cdot): V \rightarrow \mathbb{Z}$ is defined as follows: for any $v \in V$, $\operatorname{deg}(v)=$ $|\{\{v, w\}:\{v, w\} \in E\}|$ is the number of edges incident on $v$; we also define, for any $U \subseteq V$, $\operatorname{deg}(U)=\sum_{v \in U} \operatorname{deg}(v)$, i.e., the sum of degrees of the nodes contained in $U$.

6. The Jaccard similarity index is defined as follows. Given any two clusterings $\mathbf{W}_{1}, \mathbf{W}_{2}$ define

$a_{11}=$ "num. of node pairs $\{u, v\}$ in same cluster under $\mathbf{W}_{1}$ and same cluster under $\mathbf{W}_{2}$ ";

$a_{10}=$ "num. of node pairs $\{u, v\}$ in same cluster under $\mathbf{W}_{1}$ and different cluster under $\mathbf{W}_{2}$ "; $a_{01}=$ "num. of node pairs $\{u, v\}$ in different cluster under $\mathbf{W}_{1}$ and same cluster under $\mathbf{W}_{2}$ ".

Then the Jaccard similarity index $S\left(\mathbf{W}_{1}, \mathbf{W}_{2}\right)$ is defined by

$$
S\left(\mathbf{W}_{1}, \mathbf{W}_{2}\right)=\frac{a_{11}}{a_{10}+a_{01}+a_{11}} .
$$

$S\left(\mathbf{W}_{1}, \mathbf{W}_{2}\right)$ takes values in $[0,1]$; values close to 1 show that $\mathbf{W}_{1}, \mathbf{W}_{2}$ are very similar; values close to 0 that they are very different.

\section{An Intepretation of Modularity}

\subsection{Modularity}

Given a graph $G=(V, E)$ with adjacency matrix $A$, we denote the modularity of a clustering $\mathbf{V}$ by $Q_{N}(\mathbf{V}, G)$ and, following [18], we define it by

$$
Q_{N}(\mathbf{V}, G)=\frac{1}{2 m} \sum_{i, j \in V}\left(A_{i j}-\frac{\operatorname{deg}(i) \operatorname{deg}(j)}{2 m}\right) \Delta(i, j),
$$

where $\Delta(i, j)$ equals one if $i$ and $j$ belong to the same cluster and zero otherwise. Our notation emphasizes that $Q_{N}(\mathbf{V}, G)$ is a function of both the graph and the clustering. 
The motivation for introducing modularity can be seen by the following interpretation: $Q_{N}(\mathbf{V}, G)$ measures the fraction of intracluster edges in $G$ minus the expected value of the same quantity in a graph $G^{\prime}$ with the same clusters but random connections between the nodes 1 . $G^{\prime}$ is often called the null model. Note that the intracluster edge fraction of both $G$ and $G^{\prime}$ is computed with respect to $\mathbf{V}$. A large value of $Q_{N}(\mathbf{V}, G)$ indicates that, with respect to $\mathbf{V}, G$ is quite different from the null model; this is taken as evidence of $G$ having "strong community structure" which is "well captured" by V. Hence modularity is a clustering quality function $(\mathrm{CQF})$ in the sense of [9].

Other interpretations of modularity are possible; we will propose one a little later. But first let us note that, in addition to characterizing a single $(\mathbf{V}, G)$ pair, modularity can be used to compare clusterings: by definition, $\mathbf{V}$ is a better clustering of $G$ than $\mathbf{V}^{\prime}$ iff $Q_{N}(\mathbf{V}, G)>$ $Q_{N}\left(\mathbf{V}^{\prime}, G\right)$. Taking this one step further, $\mathbf{V}^{*}=\arg \max _{\mathbf{V}} Q_{N}(\mathbf{V}, G)$ is the best clustering of $G$. This has two implications: first, a large value of $\max _{\mathbf{V}} Q_{N}(\mathbf{V}, G)$ indicates that $G$ has strong community structure and second, modularity maximization can be used to obtain graph clusterings, i.e., perform community detection; this has been the basis of a large number of community detection algorithms.

While modularity maximization is a very popular method for community detection, it also has shortcomings which have been widely reported in the literature. For example, the modularity resolution limit has attracted a lot of attention [10, 11]; we will discuss it in Section 3.3. But first let us note what appears to be a more basic limitation of modularity. As already mentioned, a large $Q_{N}(\mathbf{V}, G)$ value indicates strong community structure and good clustering; but what is a "large $Q_{N}(\mathbf{V}, G)$ value"? While it is known [5] that $-\frac{1}{2} \leq Q_{N}(\mathbf{V}, G) \leq 1$ for every pair $(\mathbf{V}, G)$, examples appear in the community detection literature [9] which have strong (intuitively perceived) community structure and yet their maximum modularity is closer to zero than to one. Graphs of high modularity and weak community structure have also been reported [3, 9].

A frequently proposed explanation for the shortcomings of modularity is that the use of the null model is not well justified [9]. In Section 3.3 we will consider an alternative, complementary explanation. But first we will examine another CQF.

\subsection{Intracluster Edge Fraction}

A popular characterization of a graph community is that "there must be more edges 'inside' the community than edges linking vertices of the community with the rest of the graph" [9, Section III-B.1]. Variations of this principle have been stated by several authors ${ }^{2}$.

A prima facie reasonable way to quantify the principle is through the intracluster edge fraction, denoted by $Q_{f}(\mathbf{V}, G)$ and defined by

$$
Q_{f}(\mathbf{V}, G)=\frac{\sum_{k=1}^{K}\left|E_{k}\right|}{m} .
$$

For every $G$ and $\mathbf{V}, Q_{f}(\mathbf{V}, G) \in[0,1]$. A high (i.e., close to 1 ) value of $Q_{f}(\mathbf{V}, G)$ indicates that the pair $(\mathbf{V}, G)$ has many intracluster and few extracluster edges.

\footnotetext{
${ }^{1}$ This is a paraphrase of Newman and Girvan's description of modularity [18, Section IV].

${ }^{2}$ An extreme statement of this idea appears in [6] : "a community network $G_{0}=\left(V, E_{0}\right)$ [is] a graph $G_{0}$ that is a disjoint union of complete subgraphs".
} 
Unfortunately, a high $Q_{f}(\mathbf{V}, G)$ value does not guarantee either that $G$ has strong community structure or that $\mathbf{V}$ is a good clustering of $G$. Indeed we can always achieve the maximum value $Q_{f}(\mathbf{V}, G)=1$ by taking $\mathbf{V}=\{V\}$ (i.e., the unique clustering of size one) but this tells us nothing about the "true" community structure of $G$. This observation can be generalized. First define the following function

$$
F_{G}(K)=\max _{\mathbf{V} \in \mathcal{V}_{K}} Q_{f}(\mathbf{V}, G) .
$$

In words, for a given graph $G, F_{G}(K)$ is the maximum intracluster edge fraction achieved by clusterings of size $K$. Now we can prove the following.

Theorem 3.1 For any graph $G=(V, E), F_{G}(K)$ is a nonincreasing function of $K$.

Proof. There exists a single clustering of size one, namely $\mathbf{V}^{(1)}=\{V\}$. Denote the set of intracluster edges by $E_{1}^{(1)}$; obviously $E_{1}^{(1)}=E$ (i.e., all edges are intracluster). Hence $F_{G}(1)=$ $\frac{\left|E_{1}^{(1)}\right|}{|E|}=1$.

Let $\mathbf{V}^{(K)}=\left\{V_{1}^{(K)}, V_{2}^{(K)}, \ldots, V_{K}^{(K)}\right\}$ be the optimal clustering of size $K$; the intracluster edge sets are $E_{1}^{(K)}, \ldots, E_{K}^{(K)}$. Create a clustering $\mathbf{V}^{\prime}$ of size $K-1$ by merging $V_{K-1}^{(K)}$ and $V_{K}^{(K)}$. In other words

$$
\mathbf{V}^{\prime}=\left\{V_{1}^{(K)}, V_{2}^{(K)}, \ldots, V_{K-2}^{(K)}, V_{K-1}^{(K)} \cup V_{K}^{(K)}\right\}
$$

Under $\mathbf{V}^{\prime}$ the intracluster edges are

$$
E_{1}^{\prime}=E_{1}^{(K)}, \quad \ldots, \quad E_{K-2}^{\prime}=E_{K-2}^{(K)}, \quad E_{k-1}^{\prime} .
$$

We have $E_{K-1}^{(K)} \cup E_{K}^{(K)} \subseteq E_{K-1}^{\prime}$ and $\left|E_{K-1}^{(K)}\right|+\left|E_{K}^{(K)}\right| \leq\left|E_{K-1}^{\prime}\right|$. Hence

$$
F_{G}(K)=Q_{f}\left(\mathbf{V}^{(K)}, G\right)=\frac{\sum_{k=1}^{K}\left|E_{k}^{(K)}\right|}{|E|} \leq \frac{\sum_{k=1}^{K-2}\left|E_{k}^{(K)}\right|}{|E|}+\frac{\left|E_{K-1}^{\prime}\right|}{|E|}=Q_{f}\left(\mathbf{V}^{\prime}, G\right) .
$$

But

$$
Q_{f}\left(\mathbf{V}^{\prime}, G\right) \leq \max _{\mathbf{V} \in \mathcal{V}_{K}} Q_{f}(\mathbf{V}, G)=F_{G}(K-1) .
$$

It follows that $0 \leq F_{G}(n) \leq \ldots \leq F_{G}(2) \leq F_{G}(1)=1$ and the proof is complete.

Hence, for any $G, Q_{f}(\mathbf{V}, G)$ is maximized at $K=1$ and this gives us no information about the actual community structure of $G$. In other words, Theorem 3.1 implies that $Q_{f}$ maximization cannot determine the optimal number of clusters. On the other hand, if $K$ is given in advance (as a parameter) then $\mathbf{V}^{(K)}=\arg \max _{\mathbf{V} \in \mathcal{V}_{K}} Q_{f}(\mathbf{V}, G)$ is a reasonable candidate for the best clustering of size $K$. This has sometimes been phrased as a criticism of community detection by $Q_{f}$ maximization. For instance, in [9] is stated that "Algorithms for graph partitioning are not good for community detection, because it is necessary to provide as input the number of groups". However, this criticism is valid only to the extent that other algorithms exist which can obtain the true number of groups (clusters). For example, an alleged advantage of modularity is that its maximization yields the correct number of clusters; let us now discuss this claim. 


\subsection{Modularity as Augmented Intracluster Edge Fraction}

The claim that modularity maximization can determine the true number of clusters has been put in doubt by the discovery of the modularity resolution limit. As explained in [10, 11] and several other papers, there exist graphs $G$ for which the clustering obtained by maximizing modularity has fewer clusters than the "intuitively correct" clustering of $G$. In other words, modularity maximization can underestimate the number of clusters. We will now argue that modularity maximization can also overestimate the number of clusters. Our argument is intuitive, but it will form the basis of some precise results presented in Section 4 .

Modularity can be computed by the formula (which is known to be equivalent to (1) ):

$$
Q_{N}(\mathbf{V}, G)=\sum_{k=1}^{K} \frac{\left|E_{k}\right|}{m}-\sum_{k=1}^{K}\left(\frac{\operatorname{deg}\left(V_{k}\right)}{2 m}\right)^{2} .
$$

Defining

$$
Q_{0}(\mathbf{V}, G)=\sum_{k=1}^{K}\left(\frac{\operatorname{deg}\left(V_{k}\right)}{2 m}\right)^{2}
$$

we can rewrite (4) as

$$
Q_{N}(\mathbf{V}, G)=Q_{f}(\mathbf{V}, G)-Q_{0}(\mathbf{V}, G) .
$$

Hence Newman's modularity is the difference of $Q_{f}(\mathbf{V}, G)$ and the auxiliary function $Q_{0}(\mathbf{V}, G)$. As already mentioned, the introduction of $Q_{0}(\mathbf{V}, G)$ is usually motivated by appeal to the null model [18]; we will now present an alternative, complementary view.

Suppose momentarily that $K$ is given and we want to minimize $Q_{0}(\mathbf{V}, G)$ with respect to $\mathbf{V}=\left\{V_{1}, \ldots, V_{K}\right\}$. For simplicity of notation, define $p_{k}=\frac{\operatorname{deg}\left(V_{k}\right)}{2 m}$; then

$$
Q_{0}(\mathbf{V}, G)=\sum_{k=1}^{K}\left(\frac{\operatorname{deg}\left(V_{k}\right)}{2 m}\right)^{2}=\sum_{k=1}^{K} p_{k}^{2}
$$

and we also have

$$
\sum_{k=1}^{K} p_{k}=\sum_{k=1}^{K} \frac{\operatorname{deg}\left(V_{k}\right)}{2 m}=1
$$

Hence we want to solve the following problem:

$$
\text { given } K \text {, minimize } \sum_{k=1}^{K} p_{k}^{2} \quad \text { subject to: } 0 \leq p_{k} \leq 1 \text { and } \sum_{k=1}^{K} p_{k}=1 \text {. }
$$

Of course there are additional constraints on the $p_{k}$ 's: each of them must be obtained by summing the degrees of $V_{k}$, which is a set of nodes of the given graph $G$. However, assume for the time being that the $p_{k}$ 's are continuously valued and must only satisfy the constraints of (7) (these assumptions will be removed a little later). Under these assumptions, the solution to (7) is $p_{k}=\frac{1}{K}$ for all $k$; the minimum thus achieved is $\frac{1}{K}$.

Next consider the problem:

$$
\text { minimize } \sum_{k=1}^{K} p_{k}^{2} \quad \text { subject to: } K \in\{1, \ldots, n\}, 0 \leq p_{k} \leq 1 \text { and } \sum_{k=1}^{K} p_{k}=1 \text {. }
$$


We can solve (8) by first solving (7) separately for each $K \in\{1, \ldots, n\}$ and then looking for the overall minimum; we see that this is $\frac{1}{n}$ and is achieved at $K=n$ and $p_{k}=\frac{1}{n}$ for all $k$.

Going back to the minimization of $Q_{0}(\mathbf{V}, G)$ we note that, in general, the overall minimum $\sum_{k=1}^{K} p_{k}^{2}=\frac{1}{n}$ will only be achieved under very special circumstances. Namely, if all nodes of $\mathbf{G}$ have equal degree, then

$$
\min _{\mathbf{V} \in \mathcal{V}} Q_{0}(\mathbf{V}, G)=Q_{0}\left(\mathbf{V}^{*}, G\right)=\frac{1}{n}
$$

where $\mathbf{V}^{*}=\left\{V_{1}, \ldots, V_{n}\right\}$ and $V_{i}=\{i\}$ for $i \in\{1, \ldots, n\}$. But even when the nodes of $G$ do not have equal degrees, it seems intuitively obvious that small values of $Q_{0}(\mathbf{V}, G)$ are achieved by clusterings $\mathbf{V}$ which have many clusters (large $K$ ) and distribute nodes between clusters so that $p_{k}=\frac{\operatorname{deg}\left(V_{k}\right)}{2 m}$ is approximately the same for all $k \in\{1, \ldots, K\}$. In Section 4 we will see precise examples which justify this intuition.

Let us now apply the above observations to modularity maximization. Since (i) $Q_{N}=$ $Q_{f}-Q_{0}$, (ii) $Q_{f}$ achieves its maximum at $K=1$ and (iii) we expect $Q_{0}$ to achieve its minimum at or near $K=n$, we conclude that the following factors will influence the outcome of modularity maximization: the $Q_{f}$ term pulls $K$ towards small values and the $Q_{0}$ towards large ones; in addition the $Q_{f}$ term favors clusterings which correspond to the "natural" community structure of $G$ (i.e., there exist few extracluster edges) while the $Q_{0}$ favors "balanced" clusterings (i.e., each cluster has more or less the same degree). The final outcome depends on (among other factors) the relative magnitudes of $Q_{f}$ and $Q_{0}$.

These observations agree with previously published remarks, e.g., that "the existing modularity optimization method does not perform well in the presence of unbalanced community structures" [27] and "for modularity's null model graphs, the modularity maximum corresponds to an equipartition of the graph" [9]. However, the above works (and many other) concentrate on examples in which modularity maximization underestimates the cluster number, while our analysis suggests an overestimation effect. Since, to the best of our knowledge, overestimation examples do not appear in the literature, we will present some in Section 4.

Let us note, in concluding this section, that one method used to address the modularity resolution limit is to introduce a modified modularity function. This function is often written in the form

$$
Q(\mathbf{V}, G ; \gamma)=Q_{f}(\mathbf{V}, G)-\gamma Q_{0}(\mathbf{V}, G)
$$

where $\gamma$ is a "tuning parameter" (see [2, 14, 19, 25, 26] and also [15, 16]). With $\gamma=1$, $Q(\mathbf{V}, G ; 1)=Q_{N}(\mathbf{V}, G)$, the original Newman's modularity. If this underestimates (resp. overestimates) the "true" number of clusters, formation of more (resp. fewer) clusters can be encouraged by increasing (resp. decreasing) $\gamma$ and hence the influence of the $Q_{0}(\mathbf{V}, G)$ term on the maximization problem. However, it seems that no "universally correct" value of $\gamma$ exists; in other words, the resolution limit can occur for any $\gamma$ value [25, 26].

\section{Bad Clusterings with High Modularity}

In this section we construct graphs admitting (i) a "natural" clustering and (ii) a sequence of "arbitrarily bad" clusterings which achieve higher modularity than the natural one. In fact, as we will see, the arbitrarily bad clusterings can achieve modularity arbitrarily close to one and they can be "arbitrarily different" from the natural clustering (we will presently explain 
precisely what we mean by the terms "natural", "arbitrarily bad" and "arbitrarily different"). These results indicate that, at least in certain cases, modularity is not a good CQF.

\subsection{First Example}

To establish the abovementioned results, we will construct a family of graphs $G_{K, N_{1}, N_{2}}$ (where $K, N_{1}, N_{2}$ are parameters) such that the graph $G_{K, N_{1}, N_{2}}$ has an easily recognized "natural" clustering $\mathbf{V}_{K, N_{1}, N_{2}}$ (for every $K, N_{1}, N_{2}$ ).

We define $G_{K, N_{1}, N_{2}}$ as follows. First, for any $N_{1}, N_{2}$ we define the disconnected graph $G_{N_{1}, N_{2}}$ to be the union of a path of $N_{1}$ nodes and a path of $N_{2}$ nodes; second, we let the disconnected graph $G_{K, N_{1}, N_{2}}$ be the union of $K$ copies of $G_{N_{1}, N_{2}}$. The construction is illustrated in Figure 1 .
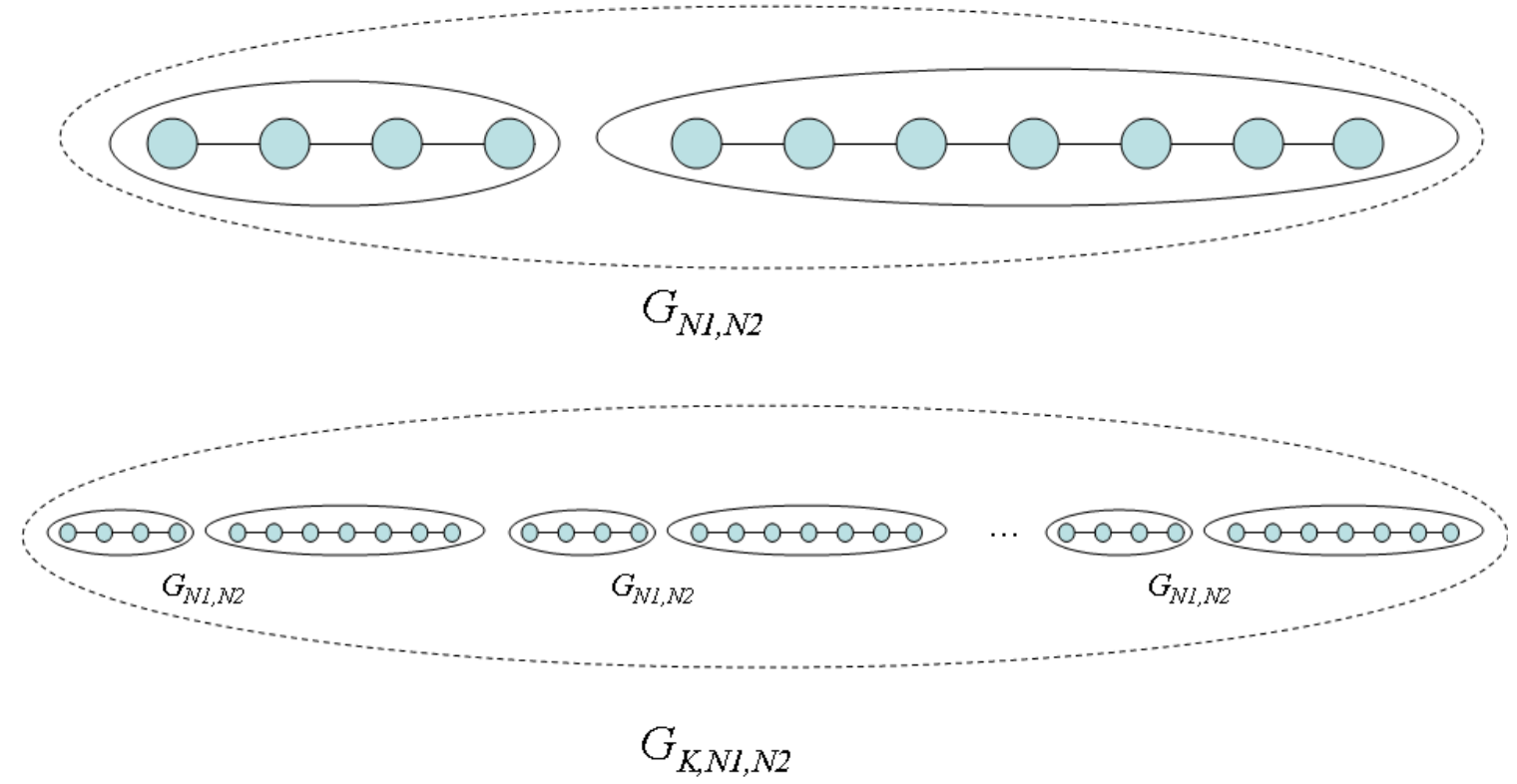

Figure 1: Graph Family $G_{K, N_{1}, N_{2}}$.

We claim that the natural clustering of $G_{K, N_{1}, N_{2}}$ is $\mathbf{V}_{K, N_{1}, N_{2}}=\left\{V_{K, N_{1}, N_{2}, 1}, V_{K, N_{1}, N_{2}, 2}, \ldots\right.$, $V_{K, N_{1}, N_{2}, 2 K}$, where $V_{K, N_{1}, N_{2}, k}$ is the node set of the $k$-th connected component of $G$ (with $k \in$ $\{1,2, \ldots, 2 K\}$, see Figure 1 . At the risk of belaboring the obvious, we note that, if $u \in V_{K, N_{1}, N_{2}, i}$ and $v \in V_{K, N_{1}, N_{2}, j}$ and $i \neq j$, then there exists no path connecting $u$ and $v$; hence they should never be put in the same cluster. So the biggest possible clusters are the $V_{K, N_{1}, N_{2}, i}$ 's. On the other hand, there is no justification for splitting some $V_{K, N_{1}, N_{2}, i}$ at any particular edge, since all edges have the same connectivity pattern, i.e., the $i$-th edge connects nodes $i$ and $i+1$. Hence $\mathbf{V}_{K, N_{1}, N_{2}}$ is the "intuitively best" (i.e., the "natural") clustering of $G_{K, N_{1}, N_{2}}$. 
Lemma 4.1 For every $K, N_{1}, N_{2} \in \mathbb{N}$ with $N_{1}, N_{2} \geq 3$ and $J \leq n=K\left(N_{1}+N_{2}\right)$ we have

$$
Q_{N}\left(\mathbf{V}_{K, N_{1}, N_{2}}, G_{K, N_{1}, N_{2}}\right)=1-\frac{\left(N_{1}-1\right)^{2}+\left(N_{2}-1\right)^{2}}{K\left(N_{1}+N_{2}-2\right)^{2}} .
$$

Proof. We fix $K, N_{1}, N_{2}$ and, for brevity, we write $G$ for $G_{K, N_{1}, N_{2}}$ and $\mathbf{V}$ for $\mathbf{V}_{K, N_{1}, N_{2}}$. We have

$$
Q_{N}(\mathbf{V}, G)=\frac{\sum_{k=1}^{2 K}\left|E_{k}\right|}{m}-\frac{\sum_{k=1}^{2 K}\left(\operatorname{deg}\left(V_{k}\right)\right)^{2}}{(2 m)^{2}}
$$

Under V, $G$ has no extracluster edges hence we have

$$
\frac{\sum_{k=1}^{2 K}\left|E_{k}\right|}{m}=1
$$

We can separate $\mathbf{V}$ into two subsets of clusters: $\mathbf{V}^{\prime}=\left\{V_{1}, V_{3}, \ldots, V_{2 K-1}\right\}$ contains the the clusters with $N_{1}$ nodes and $\mathbf{V}^{\prime \prime}=\left\{V_{2}, V_{4}, \ldots, V_{2 K}\right\}$ contains the the clusters with $N_{2}$ nodes. Each $V_{k} \in \mathbf{V}^{\prime}$ has $N_{1}-2$ "inner nodes" of degree 2 and two "border nodes" of degree 1 ; similarly, each $V_{k} \in \mathbf{V}^{\text {" }}$ has $N_{2}-2$ inner nodes and 2 border nodes. Hence

$$
\begin{aligned}
& \forall: V_{k} \in \mathbf{V}^{\prime}: \operatorname{deg}\left(V_{k}\right)=2\left(N_{1}-2\right)+2=2\left(N_{1}-1\right) \\
& \forall: V_{k} \in \mathbf{V}^{\prime \prime}: \operatorname{deg}\left(V_{k}\right)=2\left(N_{2}-2\right)+2=2\left(N_{2}-1\right)
\end{aligned}
$$

The total number of edges is

$$
m=\frac{\sum_{V_{k} \in \mathbf{V}} \operatorname{deg}\left(V_{k}\right)}{2}=\frac{\sum_{V_{k} \in \mathbf{V}^{\prime}} \operatorname{deg}\left(V_{k}\right)+\sum_{V_{k} \in \mathbf{V}^{\prime \prime}} \operatorname{deg}\left(V_{k}\right)}{2}=K\left(N_{1}+N_{2}-2\right) .
$$

Also,

$$
\begin{aligned}
\frac{\sum_{k=1}^{2 K}\left(\operatorname{deg}\left(V_{k}\right)\right)^{2}}{(2 m)^{2}} & =\frac{\sum_{V_{k} \in \mathbf{V}^{\prime}}\left(2\left(N_{1}-1\right)\right)^{2}}{\left(2 K\left(N_{1}+N_{2}-2\right)\right)^{2}}+\frac{\sum_{V_{k} \in \mathbf{V}^{\prime \prime}}\left(2\left(N_{2}-1\right)\right)^{2}}{\left(2 K\left(N_{1}+N_{2}-2\right)\right)^{2}} \\
& =\frac{K\left(N_{1}-1\right)^{2}+K \cdot\left(N_{2}-1\right)^{2}}{K^{2}\left(N_{1}+N_{2}-2\right)^{2}}=\frac{\left(N_{1}-1\right)^{2}+\left(N_{2}-1\right)^{2}}{K\left(N_{1}+N_{2}-2\right)^{2}} .
\end{aligned}
$$

Combining (10) and (11) we get (9).

Let us now introduce the "bad clusterings". For every triple $\left(K, N_{1}, N_{2}\right)$, we define a sequence $\left\{\mathbf{U}_{K, N_{1}, N_{2}, J}\right\}_{J=1}^{n}$ of clusterings of $G_{K, N_{1}, N_{2}}$. For a fixed $J$, let $L=\left\lfloor\frac{n}{J}\right\rfloor$; writing for brevity $\mathbf{U}_{J}$ in place of $\mathbf{U}_{K, N_{1}, N_{2}, J}$, we let $\mathbf{U}_{J}=\left\{U_{1}, \ldots, U_{J}, U_{J+1}\right\}$ consist of the following $J+1$ clusters:

$U_{1}=\{1, \ldots, L\}, U_{2}=\{L+1, \ldots, 2 L\}, \ldots, U_{J}=\{(J-1) L+1, \ldots, J L\}, U_{J+1}=\{J L+1, \ldots, n\} ;$

if $n=J L$ then $U_{J+1}=\emptyset$. In other words, $\mathbf{U}_{J}$ contains $J$ clusters each containing the same number of nodes (namely $L=\left\lfloor\frac{n}{J}\right\rfloor$ ) and perhaps an additional cluster (with fewer than $L$ nodes). Obviously $\mathbf{U}_{J}$ is a "well balanced" clustering.

Lemma 4.2 For every $K, N_{1}, N_{2}, J \in \mathbb{N}$ with $N_{1}, N_{2} \geq 3$ we have

$$
Q_{N}\left(\mathbf{U}_{K, N_{1}, N_{2}, J}, G_{K, N_{1}, N_{2}}\right) \geq 1-\frac{1}{K\left(N_{1}+N_{2}-2\right)} J-\frac{2\left(N_{1}+N_{2}\right)^{2}}{\left(N_{1}+N_{2}-2\right)^{2}} J^{-1} .
$$


Proof. We write $G$ for $G_{K, N_{1}, N_{2}}$ and $\mathbf{U}_{J}$ for $\mathbf{U}_{K, N_{1}, N_{2}, J}$. We have

$$
Q_{N}\left(\mathbf{U}_{J}, G\right)=\frac{\sum_{k=1}^{J+1}\left|E_{k}\right|}{m}-\frac{\sum_{k=1}^{J+1}\left(\operatorname{deg}\left(U_{k}\right)\right)^{2}}{(2 m)^{2}} .
$$

Consider first $\frac{\sum_{k=1}^{J+1}\left|E_{k}\right|}{m}$. A little thought shows that $\mathbf{U}_{J}$ has at most $J+1$ clusters and $J$ extracluster edges. Hence

$$
\forall J: \frac{\sum_{k=1}^{J+1}\left|E_{k}\right|}{m} \geq \frac{m-J}{m}=1-\frac{J}{m}=1-\frac{1}{K\left(N_{1}+N_{2}-2\right)} J .
$$

Consider now $\frac{\sum_{k=1}^{J+1}\left(\operatorname{deg}\left(U_{k}\right)\right)^{2}}{(2 m)^{2}}$. Each $U_{k}$ has no more than $\frac{n}{J}=\frac{K\left(N_{1}+N_{2}\right)}{J}$ nodes and each node has degree at most 2. Hence

$$
\forall J: \frac{\sum_{k=1}^{J+1}\left(\operatorname{deg}\left(U_{k}\right)\right)^{2}}{(2 m)^{2}} \leq \frac{(J+1) \cdot\left(2 \frac{K\left(N_{1}+N_{2}\right)}{J}\right)^{2}}{4 K^{2}\left(N_{1}+N_{2}-2\right)^{2}} \leq \frac{2\left(N_{1}+N_{2}\right)^{2}}{\left(N_{1}+N_{2}-2\right)^{2}} J^{-1}
$$

(since $\forall J \in \mathbb{N}: \frac{J+1}{J} \leq 2$ ). Combining $(13)$ and $(14)$ we get 12).

Hence, to ensure $Q_{N}\left(\mathbf{U}_{K, N_{1} N_{2}, J}, G_{K, N_{1} N_{2}}\right)>Q_{N}\left(\mathbf{V}_{K, N_{1} N_{2}}, G_{K, N_{1} N_{2}}\right)$ (i.e., that the natural clustering $\mathbf{V}_{K, N_{1} N_{2}}$ has lower modularity than $\left.\mathbf{U}_{K, N_{1} N_{2}, J}\right)$ it suffices to select $K, N_{1}, N_{2}, J$ appropriately and use Lemmas 4.1 and 4.2. A sufficient condition, obtained from (9) and (12), is

$$
1-\frac{1}{K\left(N_{1}+N_{2}-2\right)} J-\frac{2\left(N_{1}+N_{2}\right)^{2}}{\left(N_{1}+N_{2}-2\right)^{2}} J^{-1}>1-\frac{\left(N_{1}-1\right)^{2}+\left(N_{2}-1\right)^{2}}{K\left(N_{1}+N_{2}-2\right)^{2}} .
$$

Inspecting (15) we see that one way to satisfy it is by fixing $N_{1}$ and letting $J$ be "sufficiently larger" than $K$ and $N_{2}$ "sufficiently larger" than $J$. This is the main idea used in the proof of the following theorem.

Theorem 4.3 For every $K \in \mathbb{N}$ and $\varepsilon \in\left(0, \frac{1}{2 K}\right)$ there exist $N_{1}, N_{2}, J \in \mathbb{N}$ (depending on $\varepsilon$ and K) such that

$$
\begin{aligned}
& Q_{N}\left(\mathbf{V}_{K, N_{1}, N_{2}}, G_{K, N_{1}, N_{2}}\right)<1-\frac{1}{2 K}<1-\varepsilon<Q_{N}\left(\mathbf{U}_{K, N_{1}, N_{2}, J}, G_{K, N_{1}, N_{2}}\right), \\
& S\left(\mathbf{V}_{K, N_{1}, N_{2}}, \mathbf{U}_{K, N_{1}, N_{2}, J}\right)<\varepsilon .
\end{aligned}
$$

Proof. Take any $K$ and let $N_{1}=3, J=x K, N_{2}=x^{2} K$ (with $x \in \mathbb{N}$ ). To prove (16) note that

$$
\begin{aligned}
Q_{N}\left(\mathbf{V}_{K, N_{1}, N_{2}}, G_{K, N_{1}, N_{2}}\right) & =1-\frac{4+\left(x^{2} K-1\right)^{2}}{K\left(1+x^{2} K\right)^{2}}, \\
Q_{N}\left(\mathbf{U}_{K, N_{1}, N_{2}, J}, G_{K, N_{1}, N_{2}}\right) & \geq 1-\frac{x}{\left(1+x^{2} K\right)}-\frac{2\left(3+x^{2} K\right)^{2}}{\left(1+x^{2} K\right)^{2} x K} .
\end{aligned}
$$

Define $z=\frac{1}{x}$; then we have $x=\frac{1}{z}$ and

$$
Q_{N}\left(\mathbf{V}_{K, N_{1}, N_{2}}, G_{K, N_{1}, N_{2}}\right)=1-\frac{4+\left(x^{2} K-1\right)^{2}}{K\left(1+x^{2} K\right)^{2}}=1-\frac{4+\left((1 / z)^{2} K-1\right)^{2}}{K\left(1+(1 / z)^{2} K\right)^{2}} .
$$


We can simplify the final $Q_{N}\left(\mathbf{V}_{K, N_{1}, N_{2}}, G_{K, N_{1}, N_{2}}\right)$ expression of 18$)$ and write it as the following function

$$
f_{1}(z)=\frac{K^{3}-K^{2}+2\left(K+K^{2}\right) z^{2}+(K-5) z^{4}}{K\left(z^{2}+K\right)^{2}} .
$$

Now, $1-\frac{4+\left((1 / z)^{2} K-1\right)^{2}}{K\left(1+(1 / z)^{2} K\right)^{2}}$ has a removable singularity at $z_{0}=0$, but for every other $z \in \mathbb{R}$ it is identical to $f_{1}(z)$. We can expand $f_{1}(z)$ in a Taylor series around $z_{0}=0$ which will also hold for $Q_{N}\left(\mathbf{V}_{K, N_{1}, N_{2}}, G_{K, N_{1}, N_{2}}\right)$. Hence around $z_{0}=0$ we have

$$
Q_{N}\left(\mathbf{V}_{K, N_{1}, N_{2}}, G_{K, N_{1}, N_{2}}\right)=1-\frac{1}{K}+r_{1}(z)
$$

where $r_{1}(z)=a_{2} z^{2}+a_{3} z^{3}+\ldots$ and, from the Taylor series remainder theorem, there exists a constant $A$ such that, for $z$ close to zero, we have

$$
\left|r_{1}(z)\right|<A z^{2}
$$

Then, for large finite $x$ (and, in particular, for $x>\sqrt{2 K A}$ ) we have

$$
Q_{N}\left(\mathbf{V}_{K, N_{1}, N_{2}}, G_{K, N_{1}, N_{2}}\right)=1-\frac{4+\left(x^{2} K-1\right)^{2}}{K\left(1+x^{2} K\right)^{2}}<1-\frac{1}{K}+\frac{A}{x^{2}}<1-\frac{1}{2 K} .
$$

Similarly (with $z=\frac{1}{x}$ ) we have

$$
\begin{aligned}
Q_{N}\left(\mathbf{U}_{K, N_{1}, N_{2}, J}, G_{K, N_{1}, N_{2}}\right) & =1-\frac{x K}{K\left(1+x^{2} K\right)}-\frac{2\left(3+x^{2} K\right)^{2}}{\left(1+x^{2} K\right)^{2} x K} \\
& =1-\frac{(1 / z)}{\left(1+(1 / z)^{2} K\right)}-\frac{2\left(3+(1 / z)^{2} K\right)^{2}}{\left(1+(1 / z)^{2} K\right)^{2}(1 / z) K}
\end{aligned}
$$

Again, we can rewrite the final $Q_{N}\left(\mathbf{U}_{K, N_{1}, N_{2}, J}, G_{K, N_{1}, N_{2}}\right)$ expression of 20) as

$$
f_{2}(z)=\frac{K^{3}-3 K^{2} z+2 K^{2} z^{2}-13 K z^{3}+K z^{4}-18 z^{5}}{K\left(z^{2}+K\right)^{2}}
$$

and $1-\frac{(1 / z)}{\left(1+(1 / z)^{2} K\right)}-\frac{2\left(3+(1 / z)^{2} K\right)^{2}}{\left(1+(1 / z)^{2} K\right)^{2}(1 / z) K}$ has a removable singularity at $z_{0}=0$, but for every other $z \in \mathbb{R}$ it is identical to $f_{2}(z)$. Hence we can expand $f_{2}(z)$ in a Taylor series around $z_{0}=0$ which will also hold for $Q_{N}\left(\mathbf{U}_{K, N_{1}, N_{2}, J}, G_{K, N_{1}, N_{2}}\right)$. Hence around $z_{0}=0$ we have

$$
Q_{N}\left(\mathbf{U}_{K, N_{1}, N_{2}, J}, G_{K, N_{1}, N_{2}}\right)=1-\frac{3}{K} z+r_{2}(z)
$$

where $r_{2}(z)=b_{3} z^{3}+b_{4} z^{4}+\ldots$ and there exists a constant $B$ such that, for $z$ close to zero, we have

$$
\left|r_{2}(z)\right|<B z^{3}<B z^{2}
$$

this in turn implies that

$$
r_{2}(z)>-B z^{2} .
$$


Then, for large $x$ (and, in particular, for $x>K B$ ) we have

$$
\begin{aligned}
Q_{N}\left(\mathbf{U}_{K, N_{1}, N_{2}, J}, G_{K, N_{1}, N_{2}}\right) & =1-\frac{x K}{K\left(1+x^{2} K\right)}-\frac{2\left(3+x^{2} K\right)^{2}}{\left(1+x^{2} K\right)^{2} x K} \\
& >1-\frac{3}{K x}-\frac{B}{x^{2}}>1-\frac{4}{K x} .
\end{aligned}
$$

For any $\varepsilon \in\left(0, \frac{1}{2 K}\right)$, choose any $x>\max \left(\frac{4}{K \varepsilon}, \sqrt{2 K A}, K B\right)$; then we have $\frac{1}{2 K}>\varepsilon>\frac{4}{K x}$ which, combined with 19 and 21 , gives

$$
Q_{N}\left(\mathbf{U}_{K, N_{1}, N_{2}, J}, G_{K, N_{1}, N_{2}}\right)>1-\frac{4}{K x}>1-\varepsilon>1-\frac{1}{2 K}>Q_{N}\left(\mathbf{V}_{K, N_{1}, N_{2}}, G_{K, N_{1}, N_{2}}\right) .
$$

In short, we can satisfy $(16)$ for every $K \in \mathbb{N}$ and every $\varepsilon \in\left(0, \frac{1}{2 K}\right)$, by taking $x$ "sufficiently large" and $N_{1}=3, J=x \bar{K}, N_{2}=x^{2} K$.

We now turn to (17). Let $b$ (resp. $c$ ) be the number of node pairs in the same cluster under $\mathbf{U}_{K, N_{1}, N_{2}, J}$ (resp. under $\mathbf{V}_{K, N_{1}, N_{2}}$ ). We obviously have $b=a_{01}+a_{11} \geq a_{11}$ and $a_{10}+a_{01}+a_{11} \geq$ $a_{10}+a_{11}=c>0$. Hence

$$
S\left(\mathbf{U}_{K, N_{1}, N_{2}, J}, \mathbf{V}_{K, N_{1}, N_{2}}\right)=\frac{a_{11}}{a_{10}+a_{01}+a_{11}} \leq \frac{b}{c}
$$

We first obtain an upper bound for $b$. Since each $U_{j}$ contains no more than $L=\frac{n}{J}$ nodes , the number of node pairs that can be formed in $U_{j}$ is no more than $\frac{\left(\frac{n}{J}\right)\left(\frac{n}{J}-1\right)}{2}<\frac{n^{2} / 2}{J^{2}}$. Also, $n=K\left(N_{1}+N_{2}\right)$ so, for big $N_{2}, \frac{n^{2} / 2}{J^{2}}<\frac{\left(2 K N_{2}\right)^{2}}{J^{2}}$. There are at most $J+1$ clusters, so we have

$$
b<(J+1) \frac{\left(2 K N_{2}\right)^{2}}{J^{2}}=(x K+1) \frac{\left(2 K x^{2} K\right)^{2}}{(x K)^{2}}=4 K^{3} x^{3}+4 K^{2} x^{2} .
$$

Next we compute $c$. In $\mathbf{V}_{K, N_{1}, N_{2}}$ there exist $K$ clusters of $N_{1}=3$ nodes and each cluster has $\frac{N_{1}\left(N_{1}-1\right)}{2}=3$ node pairs; there also exist $K$ clusters of $N_{2}$ nodes and each cluster has $\frac{N_{2}\left(N_{2}-1\right)}{2}$ node pairs. We have

$$
c=3 K+K \frac{N_{2}\left(N_{2}-1\right)}{2}=3 K+K \frac{x^{2} K\left(x^{2} K-1\right)}{2}=\frac{1}{2} K^{3} x^{4}-\frac{1}{2} K^{2} x^{2}+3 K .
$$

And so we have

$$
\begin{aligned}
& 0 \leq S\left(\mathbf{U}_{K, N_{1}, N_{2}, J}, \mathbf{V}_{K, N_{1}, N_{2}}\right)<\frac{4 K^{3} x^{3}+4 K^{2} x^{2}}{\frac{1}{2} K^{3} x^{4}-\frac{1}{2} K^{2} x^{2}+3 K} \Rightarrow \\
& 0 \leq \lim _{x \rightarrow \infty} S\left(\mathbf{U}_{K, N_{1}, N_{2}, J}, \mathbf{V}_{K, N_{1}, N_{2}}\right) \leq \lim _{x \rightarrow \infty} \frac{4 K^{3} x^{3}+4 K^{2} x^{2}}{\frac{1}{2} K^{3} x^{4}-\frac{1}{2} K^{2} x^{2}+3 K}=0 .
\end{aligned}
$$

Hence, for every $\varepsilon>0$ and $x$ sufficiently large, (17) is satisfied.

We see from (16) that we can always find a clustering $\mathbf{U}_{K, N_{1}, N_{2}, J}$ which achieves higher modularity than the natural clustering $\mathbf{V}_{K, N_{1}, N_{2}}$ and, in fact, greater than $1-\varepsilon$, where $\varepsilon$ can get arbitrarily small independently of $K$. On the other hand, $Q_{N}\left(\mathbf{V}_{K, N_{1}, N_{2}}, G_{K, N_{1}, N_{2}}\right)$ is no greater 
than $1-\frac{1}{2 K}$; for small $K$ values this can be appreciably less than one. In other words, we can choose $K$ so that $G_{K, N_{1}, N_{2}}$ does not have very high "natural modularity" but its "artificial modularity" (the one achieved by the pair $\left(\mathbf{U}_{K, N_{1}, N_{2}, J}, G_{K, N_{1}, N_{2}}\right)$ ) can be arbitrarily close to one.

We see from (17) that, with respect to the Jaccard similarity criterion, $\mathbf{U}_{K, N_{1}, N_{2}, J}$ is very different from $\mathbf{V}_{K, N_{1}, N_{2}}$. We could have reached a similar conclusion in a simpler manner. Recall that the number of clusters of $\mathbf{U}_{K, N_{1}, N_{2}, J}$ is at least $J=x K$ and we can choose $x$ arbitrarily large; on the other hand, $\mathbf{V}_{K, N_{1}, N_{2}}$ has $2 K$ clusters. Intuitively, $\mathbf{U}_{K, N_{1}, N_{2}, J}$ must be very different from $\mathbf{V}_{K, N_{1}, N_{2}}$, since the ratio of their cluster number is $\frac{x}{2}$ and $x$ can become arbitrarily large (of course the Jaccard similarity index captures this fact in a more precise manner).

Let $\mathbf{V}^{*}=\arg \max _{V \in \mathcal{V}} Q_{N}\left(\mathbf{V}, G_{K, N_{1}, N_{2}}\right)$. While it is conceivable that $\mathbf{V}^{*}$ is more similar (in the Jaccard sense) to $\mathbf{V}_{K, N_{1}, N_{2}}$ than to some $\mathbf{U}_{K, N_{1}, N_{2}, J}$, this seems unlikely. In light of the remarks of Section 3.3, it is more likely that $\mathbf{V}^{*}$ will have many more clusters than $\mathbf{V}$. In other words, it appears that, for the graphs $G_{K, N_{1}, N_{2}}$, modularity maximization leads to an overestimation of the number of clusters, i.e., we have a case of modularity "over-resolution".

The bounds utilized in Lemmas 4.14 .2 and Theorem 4.3 are quite conservative. In many cases the inequality

$$
Q_{N}\left(\mathbf{V}_{K, N_{1}, N_{2}}, G_{K, N_{1}, N_{2}}\right)<Q_{N}\left(\mathbf{U}_{K, N_{1}, N_{2}, J}, G_{K, N_{1}, N_{2}}\right)
$$

is attained even when the abovementioned bounds are not satisfied. This can be seen in Table 1, which has been compiled by taking fixed $K=3, N_{1}=3$ and using several $x$ values (recall that $\left.J=x K, N_{2}=x^{2} K\right)$. The first six entries of each column list the quantities used in the proof of Theorem 4.3 and, for "sufficiently large" $x$, should form an increasing sequence, in accordance to the inequalities $(15)-(16)$ and $(19)-(21)$. This is indeed the case for $x=8$ and $x=10$; on the other hand, for $x=4$ and $x=6$ one inequality is violated (between the third and fourth row) but (22) still holds.

\begin{tabular}{|l|l|l|l|l|l|}
\hline Row no. & & $x=4$ & $x=6$ & $x=8$ & $x=10$ \\
\hline 1 & $Q_{N}\left(\mathbf{V}_{K, N_{1}, N_{2}}, G_{K, N_{1}, N_{2}}\right)$ & 0.6928 & 0.6787 & 0.6735 & 0.6711 \\
\hline 2 & $1-\frac{\left(N_{1}-1\right)^{2}+\left(N_{2}-1\right)^{2}}{K\left(N_{1}+N_{2}-2\right)^{2}}$ & 0.6928 & 0.6787 & 0.6735 & 0.6711 \\
\hline 3 & $1-\frac{1}{2 K}$ & 0.8333 & 0.8333 & 0.8333 & 0.8333 \\
\hline 4 & $1-\frac{4}{K x}$ & 0.6667 & 0.7778 & 0.8333 & 0.8667 \\
\hline 5 & $1-\frac{1}{K\left(N_{1}+N_{2}-2\right)} J-\frac{2\left(N_{1}+N_{2}\right)^{2}}{\left(N_{1}+N_{2}-2\right)^{2}} J^{-1}$ & 0.7378 & 0.8297 & 0.8735 & 0.8992 \\
\hline 6 & $Q_{N}\left(\mathbf{U}_{K, N_{1}, N_{2}, J}, G_{K, N_{1}, N_{2}}\right)$ & 0.8407 & 0.8915 & 0.9179 & 0.9340 \\
\hline 7 & $S\left(\mathbf{U}_{K, N_{1}, N_{2}, J}, \mathbf{V}_{K, N_{1}, N_{2}}\right)$ & 0.2196 & 0.1540 & 0.1190 & 0.0967 \\
\hline
\end{tabular}

Table 1. Several quantities appearing in the proof of Theorem 4.3. In each column and for rows 1 to 6 , for large enough $x$, the value of each row must be no less than that of the previous one.

From rows 1 and 6 of Table 1 we see that $Q_{N}\left(\mathbf{V}_{K, N_{1}, N_{2}}, G_{K, N_{1}, N_{2}}\right)$ is a decreasing and $Q_{N}\left(\mathbf{U}_{K, N_{1}, N_{2}, J}, G_{K, N_{1}, N_{2}}\right)$ an increasing function of $x$. From row 7 we see that the the Jaccard similarity is a decreasing function of $x$. These observations verify straightforward conclusions which can be drawn from the proof of Theorem 4.3 . 


\subsection{Second Example}

It might be argued that the results of Section 4.1 are only possible because we have used the disconnected graphs $G_{K, N_{1}, N_{2}}$. This is not the case. In this section we will illustrate the same issues using the family of connected graphs $H_{K, N_{1}, N_{2}}$ illustrated in Figure 2, We start with connected $H_{N_{1}, N_{2}}$ graphs, each of which is a path of $N_{1}+N_{2}$ nodes, with extra edges added between the first $N_{1}$ (resp. the second $N_{2}$ ) nodes at distance two of each other. Then we construct the $H_{K, N_{1}, N_{2}}$ graphs by joining in series $K H_{N_{1}, N_{2}}$ subgraphs.
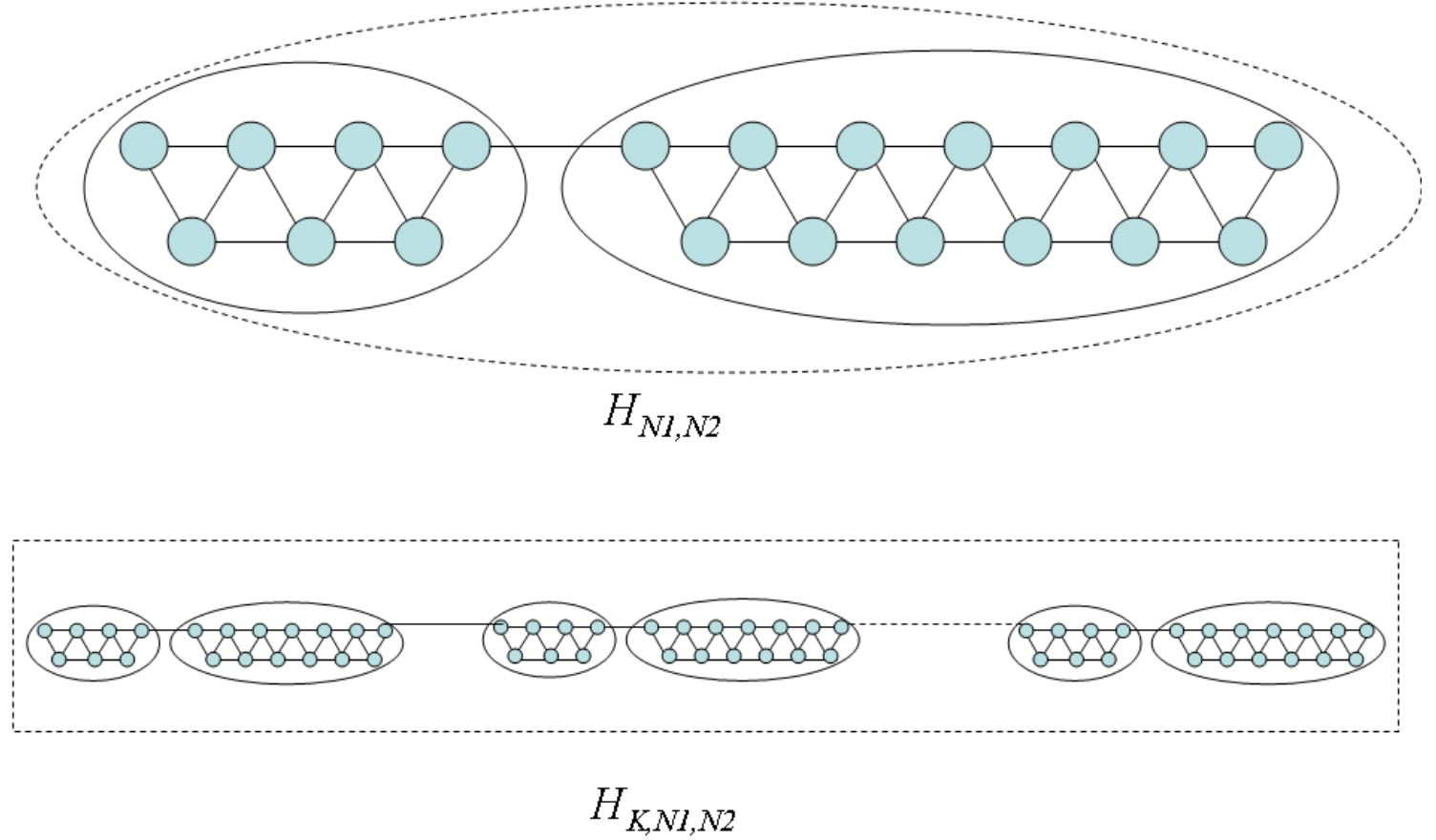

Figure 2: Graph Family $H_{K, N_{1}, N_{2}}$.

We will use the same clusterings $\mathbf{V}_{K, N_{1}, N_{2}}$ and clustering sequences $\left\{\mathbf{U}_{K, N_{1}, N_{2} J}\right\}_{J=1}^{n}$ as in Section 4.1. Once again, for reasons similar to the ones discussed in Section 4.1, we claim that $\mathbf{V}_{K, N_{1}, N_{2}}$ is the natural clustering of $H_{K, N_{1}, N_{2}}$. Namely, cluster boundaries should occur across edges incident on the most weakly connected nodes; this shows that the $V_{K, N_{1}, N_{2}, k}$ clusters must be preserved; any partition of $V_{K, N_{1}, N_{2}, k}$ into finer clusters cannot be justified, since all of its edges have the same connectivity pattern. Hence $\mathbf{V}_{K, N_{1}, N_{2}}$ is the "intuitively best" (i.e., the "natural") clustering of $H_{K, N_{1}, N_{2}}$.

Once again, we obtain (in three steps) a result similar to Theorem 4.3. First we need two lemmas. 
Lemma 4.4 For every $K, N_{1}, N_{2} \in \mathbb{N}$ with $N_{1}, N_{2} \geq 5$ we have

$$
Q_{N}\left(\mathbf{V}_{K, N_{1}, N_{2}}, H_{K, N_{1}, N_{2}}\right)<1-\frac{K\left(\left(4 N_{1}-8\right)^{2}+\left(4 N_{2}-8\right)^{2}\right)}{\left(4 K\left(N_{1}+N_{2}-2\right)\right)^{2}} .
$$

Proof. We fix $K, N_{1}, N_{2}$ and, for brevity, we write $H$ for $H_{K, N_{1}, N_{2}}$ and $\mathbf{V}$ for $\mathbf{V}_{K, N_{1}, N_{2}} ; \mathbf{V}^{\prime}$ and $\mathbf{V}^{\prime \prime}$ have the same meaning as previously. In $\mathbf{V}_{K, N_{1}, N_{2}}$ there exist $2 K-1$ extracluster edges, so we have

$$
\frac{\sum_{k=1}^{2 K}\left|E_{k}\right|}{m}<1
$$

For each $V_{k} \in \mathbf{V}^{\prime}$, there are two border nodes on the left, two border nodes on the right and $N_{1}-4$ inner nodes. Each of the inner nodes has degree 4; each of the border nodes has degree 3 , except for the first and last node of the graph, which have degree 2 . Hence for each $V_{k} \in \mathbf{V}^{\prime}$ we have the bounds

$$
\left(N_{1}-4\right) \cdot 4+4 \cdot 2=4 N_{1}-8<\operatorname{deg}\left(V_{k}\right)<4 N_{1}-4=\left(N_{1}-4\right) \cdot 4+4 \cdot 3 .
$$

Similarly, for each $V_{k} \in \mathbf{V}^{\prime \prime}$ we have the bounds

$$
\left(N_{2}-4\right) \cdot 4+4 \cdot 2=4 N_{2}-8<\operatorname{deg}\left(V_{k}\right)<4 N_{2}-4=\left(N_{2}-4\right) \cdot 4+4 \cdot 3 .
$$

The total number of edges is $m=\frac{\sum_{k=1}^{2 K} \operatorname{deg}\left(V_{k}\right)}{2}$ and we have

$$
\begin{aligned}
\frac{K\left(4 N_{1}-8+4 N_{2}-8\right)}{2} & <\frac{\sum_{k=1}^{2 K} \operatorname{deg}\left(V_{k}\right)}{2}<\frac{K\left(4 N_{1}-4+4 N_{2}-4\right)}{2} \Rightarrow \\
2 K\left(N_{1}+N_{2}-4\right) & <m<2 K\left(N_{1}+N_{2}-2\right) .
\end{aligned}
$$

In addition we have

$$
K\left(\left(4 N_{1}-8\right)^{2}+\left(4 N_{2}-8\right)^{2}\right)<\sum_{k=1}^{2 K}\left(\operatorname{deg}\left(V_{k}\right)\right)^{2}<K\left(\left(4 N_{1}-4\right)^{2}+\left(4 N_{2}-4\right)^{2}\right) .
$$

Combining (25) and (26) we get

$$
\frac{\sum_{k=1}^{2 K}\left(\operatorname{deg}\left(V_{k}\right)\right)^{2}}{(2 m)^{2}}>\frac{K\left(\left(4 N_{1}-8\right)^{2}+\left(4 N_{2}-8\right)^{2}\right)}{\left(4 K\left(N_{1}+N_{2}-2\right)\right)^{2}} .
$$

Finally, combining (24) and 27) we get the required bound.

Lemma 4.5 For every $K, N_{1}, N_{2}, J \in \mathbb{N}$ with $N_{1}, N_{2} \geq 5$ and $J \leq n=K\left(N_{1}+N_{2}\right)$ we have

$$
Q_{N}\left(\mathbf{U}_{K, N_{1}, N_{2}, J}, H_{K, N_{1}, N_{2}}\right)>1-\frac{3}{2 K\left(N_{1}+N_{2}-4\right)} J-\frac{2\left(N_{1}+N_{2}\right)^{2}}{\left(N_{1}+N_{2}-4\right)^{2}} J^{-1} .
$$


Proof. Extracluster edges in $\mathbf{U}_{J}$ can only occur between successive clusters ${ }^{3} U_{k}, U_{k+1}$; between any such pair there exist at most three such edges; hence $\mathbf{U}_{J}$ cannot have more than $3 J$ extracluster edges. Consequently

$$
\frac{\sum_{k=1}^{J+1}\left|E_{k}\right|}{m} \geq \frac{m-3 J}{m}=1-\frac{3 J}{m}>1-\frac{3 J}{2 K\left(N_{1}+N_{2}-4\right)} .
$$

Each $U_{k}$ has at most $\frac{n}{J}=\frac{K\left(N_{1}+N_{2}\right)}{J}$ nodes and each node has degree at most 4 . Hence

$$
\frac{\sum_{k=1}^{J+1}\left(\operatorname{deg}\left(U_{k}\right)\right)^{2}}{(2 m)^{2}} \leq \frac{(J+1)\left(4 \frac{K\left(N_{1}+N_{2}\right)}{J}\right)^{2}}{\left(4 K\left(N_{1}+N_{2}-4\right)\right)^{2}} \leq \frac{2\left(N_{1}+N_{2}\right)^{2}}{\left(N_{1}+N_{2}-4\right)^{2}} J^{-1} .
$$

Combining (29) and (30) we get the bound 28$)$.

To ensure $Q_{N}\left(\mathbf{U}_{K, N_{1} N_{2}, J}, H_{K, N_{1} N_{2}}\right)>Q_{N}\left(\mathbf{V}_{K, N_{1} N_{2}}, H_{K, N_{1} N_{2}}\right)$ it suffices to choose appropriate $K, N_{1}, N_{2}, J$ and use Lemmas 4.4 and 4.5. A sufficient condition, obtained from (23) and (28), is

$$
1-\frac{3}{2 K\left(N_{1}+N_{2}-4\right)} J-\frac{2\left(N_{1}+N_{2}\right)^{2}}{\left(N_{1}+N_{2}-4\right)^{2}} J^{-1}>1-\frac{K \cdot\left(\left(4 N_{1}-8\right)^{2}+\left(4 N_{2}-8\right)^{2}\right)}{\left(4 K\left(N_{1}+N_{2}-2\right)\right)^{2}} .
$$

Now we can prove the following.

Theorem 4.6 For every $K \in \mathbb{N}$ and $\varepsilon \in\left(0, \frac{1}{2 K}\right)$ there exist $N_{1}, N_{2}, J \in \mathbb{N}$ (depending on $\varepsilon, K$ ) such that

$$
\begin{aligned}
& Q_{N}\left(\mathbf{V}_{K, N_{1}, N_{2}}, H_{K, N_{1}, N_{2}}\right)<1-\frac{1}{2 K}<1-\varepsilon<Q_{N}\left(\mathbf{U}_{K, N_{1}, N_{2}, J}, H_{K, N_{1}, N_{2}}\right) \\
& S\left(\mathbf{V}_{K, N_{1}, N_{2}}, \mathbf{U}_{K, N_{1}, N_{2}, J}\right)<\varepsilon .
\end{aligned}
$$

Proof. Take any $K$. Letting $N_{1}=6, J=x K, N_{2}=x^{2} K$ we have

$$
\begin{gathered}
Q_{N}\left(\mathbf{V}_{K, N_{1}, N_{2}}, H_{K, N_{1}, N_{2}}\right)<1-\frac{K\left(16^{2}+\left(4 x^{2} K-8\right)^{2}\right)}{\left(4 K\left(4+x^{2} K\right)\right)^{2}}, \\
Q_{N}\left(\mathbf{U}_{K, N_{1}, N_{2}, J}, H_{K, N_{1}, N_{2}}\right)>1-\frac{3 x}{2\left(2+x^{2} K\right)}-\frac{2\left(6+x^{2} K\right)^{2}}{x K\left(2+x^{2} K\right)^{2}} .
\end{gathered}
$$

Defining $z=\frac{1}{x}$ we have $x=\frac{1}{z}$ and

$$
1-\frac{K\left(16^{2}+\left(4 x^{2} K-8\right)^{2}\right)}{\left(4 K\left(4+x^{2} K\right)\right)^{2}}=1-\frac{K\left(16^{2}+\left(4(1 / z)^{2} K-8\right)^{2}\right)}{\left(4 K\left(4+(1 / z)^{2} K\right)\right)^{2}} .
$$

Similarly to the proof of Theorem 4.3, there is a function $f_{3}(z)$ which, for every $z \neq z_{0}=0$, is equal to the right part of (34) and around $z_{0}$ has the Taylor expansion

$$
f_{3}(z)=1-\frac{1}{K}+r_{3}(z)
$$

\footnotetext{
${ }^{3}$ There is an exception when $J=n$, but in this case too 29 holds.
} 
where $r_{3}(z)=c_{2} z^{2}+c_{3} z^{2}+\ldots$. Furthermore, there exists a constant $C$ such that, for $z$ close to zero, we have

$$
\left|r_{3}(z)\right|<C z^{2}
$$

Then, for large $x$ (and in particular for $x>\sqrt{2 K C}$ ) we have

$$
Q_{N}\left(\mathbf{V}_{K, N_{1}, N_{2}}, H_{K, N_{1}, N_{2}}\right)<1-\frac{1}{K}+\frac{C}{x^{2}}<1-\frac{1}{2 K}
$$

Similarly, with $z=1 / x$, we have

$$
\begin{aligned}
Q_{N}\left(\mathbf{U}_{K, N_{1}, N_{2}, J}, H_{K, N_{1}, N_{2}}\right) & >1-\frac{3 x K}{2 K\left(2+x^{2} K\right)}-\frac{2\left(6+x^{2} K\right)^{2}}{x K\left(2+x^{2} K\right)^{2}} \\
& =1-\frac{3(1 / z) K}{2 K\left(2+(1 / z)^{2} K\right)}-\frac{2\left(6+(1 / z)^{2} K\right)^{2}}{(1 / z) K\left(2+(1 / z)^{2} K\right)^{2}}=f_{4}(z) .
\end{aligned}
$$

Once again, there is a function $f_{4}(z)$ which, for every $z \neq z_{0}=0$, is equal to the right part of (36) and around $z_{0}$ has the Taylor expansion

$$
f_{4}(z)=1-\frac{7}{2 K} z+r_{4}(z)
$$

where $r_{4}(z)=d_{3} z^{3}+d_{4} z^{4}+\ldots$. And there exists a constant $D$ such that, for $z$ close to zero, we have

$$
\left|r_{4}(z)\right|<D z^{3}<D z^{2}, \quad r_{4}(z)>-D z^{2} .
$$

Then, for large $x$ (an, in particular, for $x>2 K D$ ) we have

$$
\begin{aligned}
Q_{N}\left(\mathbf{U}_{K, N_{1}, N_{2}, J}, H_{K, N_{1}, N_{2}}\right) & >1-\frac{3 x K}{2 K\left(2+x^{2} K\right)}-\frac{2\left(6+x^{2} K\right)^{2}}{x K\left(2+x^{2} K\right)^{2}} \\
& >1-\frac{7}{2 K x}-\frac{D}{x^{2}}>1-\frac{4}{K x} .
\end{aligned}
$$

For any $\varepsilon \in\left(0, \frac{1}{2 K}\right)$ choose any $x>\max \left(\frac{4}{K \varepsilon}, \sqrt{2 K C}, 2 K D\right)$; then we have $\frac{1}{2 K}>\varepsilon>\frac{4}{K x}$ which, combined with (35) and (37), yields

$$
Q_{N}\left(\mathbf{U}_{K, N_{1}, N_{2}, J}, G_{K, N_{1}, N_{2}}\right)>1-\frac{4}{K x}>1-\varepsilon>1-\frac{1}{2 K}>Q_{N}\left(\mathbf{V}_{K, N_{1}, N_{2}}, G_{K, N_{1}, N_{2}}\right) .
$$

In short, we can satisfy $(32)$ for every $K \in \mathbb{N}$ and every $\varepsilon \in\left(0, \frac{1}{2 K}\right)$, by taking $x$ "sufficiently large" and $N_{1}=6, J=x \bar{K}, N_{2}=x^{2} K$.

Finally, (33) is exactly the same as $(17)$ and has already been proved.

Similarly to Section 4.1, the bounds utilized in Lemmas 4.4 4.5 and Theorem 4.6 are conservative and the inequality

$$
Q_{N}\left(\mathbf{V}_{K, N_{1}, N_{2}}, H_{K, N_{1}, N_{2}}\right)<Q_{N}\left(\mathbf{U}_{K, N_{1}, N_{2}, J}, H_{K, N_{1}, N_{2}}\right)
$$

can be satisfied even when the bounds are violated. This can be seen in Table 2, which is analogous to Table 1 of Section 4.1. We have used $K=3, N_{1}=6$ and several $x$ values. The first six entries of each column list the quantities used in the proof of Theorem 4.3 and, for "sufficiently large" $x$, should form an increasing sequence. This is the case for $x=8$ and $x=10$; for $x=6$ the sequence is not increasing but (38) holds. 


\begin{tabular}{|l|l|l|l|l|}
\hline Row no. & & $x=6$ & $x=8$ & $x=10$ \\
\hline 1 & $Q_{N}\left(\mathbf{V}_{K, N_{1}, N_{2}}, G_{K, N_{1}, N_{2}}\right)$ & 0.6872 & 0.6787 & 0.6745 \\
\hline 2 & $1-\frac{K\left(\left(4 N_{1}-8\right)^{2}+\left(4 N_{2}-8\right)^{2}\right)}{\left(4 K\left(N_{1}+N_{2}-2\right)\right)^{2}}$ & 0.7010 & 0.6866 & 0.6796 \\
\hline 3 & $1-\frac{1}{2 K}$ & 0.8333 & 0.8333 & 0.8333 \\
\hline 4 & $1-\frac{4}{K x}$ & 0.7778 & 0.8333 & 0.8667 \\
\hline 5 & $1-\frac{3}{2 K\left(N_{1}+N_{2}-4\right)} J-\frac{2\left(N_{1}+N_{2}\right)^{2}}{\left(N_{1}+N_{2}-4\right)^{2}} J^{-1}$ & 0.7988 & 0.8513 & 0.8819 \\
\hline 6 & $Q_{N}\left(\mathbf{U}_{K, N_{1}, N_{2}, J}, G_{K, N_{1}, N_{2}}\right)$ & 0.8743 & 0.8986 & 0.9182 \\
\hline 7 & $S\left(\mathbf{U}_{K, N_{1}, N_{2}, J}, \mathbf{V}_{K, N_{1}, N_{2}}\right)$ & 0.1695 & 0.1183 & 0.0956 \\
\hline
\end{tabular}

Table 2. Several quantities appearing in the proof of Theorem [4.6. In each column and for rows 1 to 6 , for large enough $x$, the value of each row must be no less than that of the previous one.

From rows 1 and 6 of Table 2 we see that $Q_{N}\left(\mathbf{V}_{K, N_{1}, N_{2}}, G_{K, N_{1}, N_{2}}\right)$ is decreasing with $x$ and $Q_{N}\left(\mathbf{U}_{K, N_{1}, N_{2}, J}, G_{K, N_{1}, N_{2}}\right)$ is increasing; from row 7 we see that the Jaccard similarity is decreasing with $x$.

\section{Discussion and Related Work}

Theorems 4.3 and 4.6 cast doubt on the efficacy of Newman's modularity $Q_{N}$ as "an objective metric for choosing the number of communities" [18]. In fact, the existence of such an objective metric can be doubted and the meaning of the terms "best clustering", "natural clustering", etc. are rather ambiguous, as noted by several researchers (for a discussion see [9, Section III]).

Consider for example the term "good clustering". A good clustering $\mathbf{V}$ of a graph $G$ should be objectively recognizable by a high value of $Q(\mathbf{V}, G)$, where $Q$ is a "good CQF". However, a good CQF is one which assigns high scores to good clusterings. Hence it appears that the definition of "good clusterings" and "good CQF" is a circular process.

While obtaining a "good CQF" is a worthwhile target, the main focus of the current paper has been the use of $Q_{N}$ towards estimation of the true number of communities. Since we have argued that $Q_{N}$ 's performance is less than ideal, let us conclude by discussing alternative ways to perform community number selection.

Let us start by stating that we consider "community number selection" to be a special case of the general problem of "cluster number selection", which has been exhaustively studied in the "classic" clustering literature (see for example the book [8]). In this literature, cluster number selection has been recognized as "a fundamental, and largely unsolved, problem in cluster analysis" [23].

Many works in the the "classic" clustering literature treat cluster number selection through a two-stage approach. First, a CQF is used to obtain the optimal clustering of size $K$, for $K \in\left\{1,2, \ldots, K_{\max }\right\}$. Then the optimal $K$ value (and hence the overall optimal clustering) is obtained using a cluster number selection criterion (CNSC) ${ }^{4}$ such as the Akaike Information Criterion [1], the Bayesian Information Criterion [22], Minimum Description Length [20], the gap statistic [24], the knee criterion [21] etc. Details on CNSC can be found in [4, 7, 12, 13, 17].

\footnotetext{
${ }^{4}$ The terms model order selection criterion and cluster validity selection criterion are also used.
} 
The two-stage approach has also been used in the community detection literature. In Newman's seminal paper [18] a dendrogram is obtained through a divisive hierarchical clustering process (which uses betweenness, rather than $Q_{N}$ ) and the dendrogram cutoff level (and hence the number of communities) is obtained by maximizaton of $Q_{N}$. In this case $Q_{N}$ is used as CNSC rather than as a CQF.

We find the two-stage approach to community detection promising and we believe it deserves further research. In particular, we expect that the two-stage approach will yield better results if a better CNSC than $Q_{N}$ is used. However the specification of good CNSC's must overcome the same difficulties (associated with circularity) mentioned in the beginning of this section. We hope these difficulties can be alleviated by the use of an axiomatic approach, which we will report in a future publication.

\section{References}

[1] H. Akaike, A new look at the statistical model identification, Automatic Control, IEEE Transactions on 19 (1974), no. 6, 716-723.

[2] J.I. Alvarez-Hamelin, B.M. Gastón, and J.R. Busch, On weakly optimal partitions in modular networks, Arxiv preprint arXiv:1008.3443 (2010).

[3] J.P. Bagrow, Communities and bottlenecks: Trees and treelike networks have high modularity, Physical Review E 85 (2012), no. 6, 066118.

[4] F. Boutin and M. Hascoet, Cluster validity indices for graph partitioning, Information Visualisation, 2004. IV 2004. Proceedings. Eighth International Conference on, IEEE, 2004, pp. 376-381.

[5] U. Brandes, D. Delling, M. Gaertler, R. Görke, M. Hoefer, Z. Nikoloski, and D. Wagner, On finding graph clusterings with maximum modularity, Graph-Theoretic Concepts in Computer Science, Springer, 2007, pp. 121-132.

[6] WYC Chen, AWM Dress, and WQ Yu, Checking the reliability of a linear-programming based approach towards detecting community structures in networks, IET systems biology 1 (2007), 286.

[7] E. Dimitriadou, S. Dolničar, and A. Weingessel, An examination of indexes for determining the number of clusters in binary data sets, Psychometrika 67 (2002), no. 1, 137-159.

[8] R.O. Duda, P.E. Hart, and D.G. Stork, Pattern classification and scene analysis 2nd ed., (1995).

[9] S. Fortunato, Community detection in graphs, Physics Reports 486 (2010), no. 3-5, 75-174.

[10] S. Fortunato and M. Barthelemy, Resolution limit in community detection, Proceedings of the National Academy of Sciences 104 (2007), no. 1, 36.

[11] B.H. Good, Y.A. de Montjoye, and A. Clauset, Performance of modularity maximization in practical contexts, Physical Review E 81 (2010), no. 4, 046106. 
[12] M. Halkidi, Y. Batistakis, and M. Vazirgiannis, Cluster validity methods: part i, ACM Sigmod Record 31 (2002), no. 2, 40-45.

[13] _ Clustering validity checking methods: part ii, ACM Sigmod Record 31 (2002), no. $3,19-27$.

[14] G. Krings and V.D. Blondel, An upper bound on community size in scalable community detection, Arxiv preprint arXiv:1103.5569 (2011).

[15] J.M. Kumpula, J. Saramäki, K. Kaski, and J. Kertesz, Limited resolution in complex network community detection with potts model approach, The European Physical Journal BCondensed Matter and Complex Systems 56 (2007), no. 1, 41-45.

[16] Z. Li, S. Zhang, R.S. Wang, X.S. Zhang, and L. Chen, Quantitative function for community detection, Physical review E 77 (2008), no. 3, 036109.

[17] G.W. Milligan and M.C. Cooper, An examination of procedures for determining the number of clusters in a data set, Psychometrika 50 (1985), no. 2, 159-179.

[18] M.E.J. Newman and M. Girvan, Finding and evaluating community structure in networks, Physical review E 69 (2004), no. 2, 026113.

[19] J. Reichardt and S. Bornholdt, Statistical mechanics of community detection, Physical Review E 74 (2006), no. 1, 016110.

[20] J. Rissanen, Modeling by shortest data description, Automatica 14 (1978), no. 5, 465-471.

[21] S. Salvador and P. Chan, Determining the number of clusters/segments in hierarchical clustering/segmentation algorithms, Tools with Artificial Intelligence, 2004. ICTAI 2004. 16th IEEE International Conference on, IEEE, 2004, pp. 576-584.

[22] G. Schwarz, Estimating the dimension of a model, The annals of statistics 6 (1978), no. 2, 461-464.

[23] C.A. Sugar and G.M. James, Finding the number of clusters in a dataset, Journal of the American Statistical Association 98 (2003), no. 463, 750-763.

[24] R. Tibshirani, G. Walther, and T. Hastie, Estimating the number of clusters in a data set via the gap statistic, Journal of the Royal Statistical Society: Series B (Statistical Methodology) 63 (2001), no. 2, 411-423.

[25] VA Traag, P. Van Dooren, and Y. Nesterov, Narrow scope for resolution-limit-free community detection, Physical Review E 84 (2011), no. 1, 016114.

[26] J. Xiang and K. Hu, Limitation of multi-resolution methods in community detection, Arxiv preprint arXiv:1108.4244 (2011).

[27] S. Zhang and H. Zhao, Community identification in networks with unbalanced structure, Physical Review E 85 (2012), no. 6, 066114. 